\title{
Efficacy of TNF $\alpha$ blockade in cyclophosphamide resistant neuro-Behçet disease
}

\author{
C Ribi, R Sztajzel, J Delavelle, C Chizzolini
}

J Neurol Neurosurg Psychiatry 2005;76:1733-1735. doi: 10.1136/jnnp.2004.055434

\begin{abstract}
Behçet disease is a chronic relapsing inflammatory condition, predominantly affecting young adults, characterised by recurrent bipolar aphtae and systemic manifestations for which tumour necrosis factor (TNF) $\alpha$ blockade has recently emerged as an effective treatment. We report the case of a patient presenting with mucocutaneous and ocular manifestations who in the course of his disease developed CNS parenchymal involvement. While being treated with pulsed cyclophosphamide and corticosteroids, he suffered a relapse of his CNS involvement that was efficaciously controlled by infliximab. No disease activity was observed during a full year of TNF blockade, associated with azathioprine, colchicine, and corticosteroids. However, 7 months after the last administration of infliximab and still under immunosuppressant agents, CNS lesions recurred. Infliximab was successfully reintroduced and since continued with no side effects. The sequence of events observed in this patient suggests that TNF blockade is efficacious in suppressing neuro-Behçet disease and once introduced should be maintained for a prolonged period of time.
\end{abstract}

\section{A} 23 year old man was diagnosed with Behçet disease (BD) on the basis of recurrent oral and genital aphtae, pustulosis, and posterior uveitis. Positivity for HLA-B51 further supported the diagnosis. Mucocutaneous and ocular relapses occurred under systemic corticosteroids and colchicine. Cyclosporine A at a daily dose of $2 \mathrm{mg} / \mathrm{kg}$ prevented further ocular involvement, and mucocutaneous flares were managed with short courses of thalidomide. Three years after disease onset, the patient stopped most medications while consuming cannabis and amphetamines and sniffing cocaine. He developed left sided headache of increasing intensity and loss of strength in the right leg and subsequently in the right arm with a concomitant flare of oral and genital ulcers. On admission, he presented with slight dysarthria and right sided motor and sensory deficits including the face. Magnetic resonance imaging (MRI) showed a high signal lesion of the left thalamus and the posterior limb of the internal capsule, extending into homolateral mesencephalon (fig 1A). Fifteen white cells/ $\mathrm{mm}^{3}$ (28\% polynuclear) were present on CSF analysis. Glucose and total protein levels were normal. Neuro-Behçet disease (NB) was diagnosed and treatment with high dose steroids and monthly cyclophosphamide pulses instituted. Colchicine and aspirin were continued. Neurological deficits partially resolved, and MRI performed 3 months later showed regression of the capsular lesion and no more signs of disease in the thalamo-mesencephalic region. Four months and five cyclophosphamide pulses after the initial episode, under a daily prednisone dose of $20 \mathrm{mg}$, the patient relapsed. Aphasia and right sided hemiparesis worsened acutely and MRI disclosed new lesions located in the left sided temporal lobe and reappearance of the lesion in the left sided mesencephalon (fig 1B). CSF analysis revealed a predominantly polynuclear pleocytosis with 400 cells $/ \mathrm{mm}^{3}$, moderate elevation of total protein, and normal glucose levels. An infectious complication in an immunocompromised patient was excluded. Pulsed methylprednisolone was administered and intravenous cyclophosphamide continued. Intravenous infliximab was administered at a dose of $5 \mathrm{mg} / \mathrm{kg}$ and repeated after 2 and 6 weeks. Cerebral MRI studies showed rapid regression of the acute lesions and partial clinical improvement was progressively noted. Infliximab was continued bimonthly for 1 year, while cyclophosphamide was replaced by azathioprine after 6 months. Colchicine was continued and prednisone tapered to $10 \mathrm{mg} / \mathrm{day}$. Seven months after the last administration of infliximab, while on azathioprine, colchicine, and prednisone, the patient presented with headache, worsening of speech, and right sided hemiparesis. MRI showed new contrast enhancement in the left capsula interna (fig $1 C$ ). The patient responded well to high dose corticosteroids and a new infliximab infusion. Since then, tumour necrosis factor (TNF) blockade has been given bimonthly and is well tolerated. Right sided motor and sensory deficit and neuropsychological difficulties, present since the first episode and exacerbated by subsequent relapses, persist. There are no clinical or radiological signs of disease activity 16 months after the last relapse (figs 1D and 2).

\section{DISCUSSION}

In BD, male gender, young age, and the presence of HLA-B5 I antigen are associated with more aggressive disease course. Indeed, our patient presented with severe BD, characterised by early ocular and CNS involvement and poor response to conventional treatment. Diagnosis of recurrent meningoencephalitis was retained on the basis of typical MRI and CSF features.

The reported incidence of neurological involvement in BD varies greatly, probably due to selection bias. In 3050 nonselected Turkish patients with $\mathrm{BD}$, the reported incidence of NB was $4.8 \%$ with clear male preponderance and with a mean age at $\mathrm{BD}$ onset of 26.7 years and 32.0 years at the time of neurological involvement. ${ }^{1}$ Relapses are common and some patients develop a progressive disease course. Headache is the most prevalent symptom in NB and should prompt further investigations. Meningoencephalitis with brainstem involvement, followed by spinal cord and hemisphere involvement, are the most frequent clinical presentations. ${ }^{2}$ Cranial neuropathy and subacute encephalopathy are rarer. Cerebral venous sinus thrombosis is frequent, produces limited symptoms, has better outcome, and usually is mutually exclusive with parenchymal NB. MRI is the imaging study of

Abbreviations: $B B B$, blood-brain barrier; BD, Behçet disease; MRI, magnetic resonance imaging; NB, neuro-Behçet disease; TNF, tumour necrosis factor 

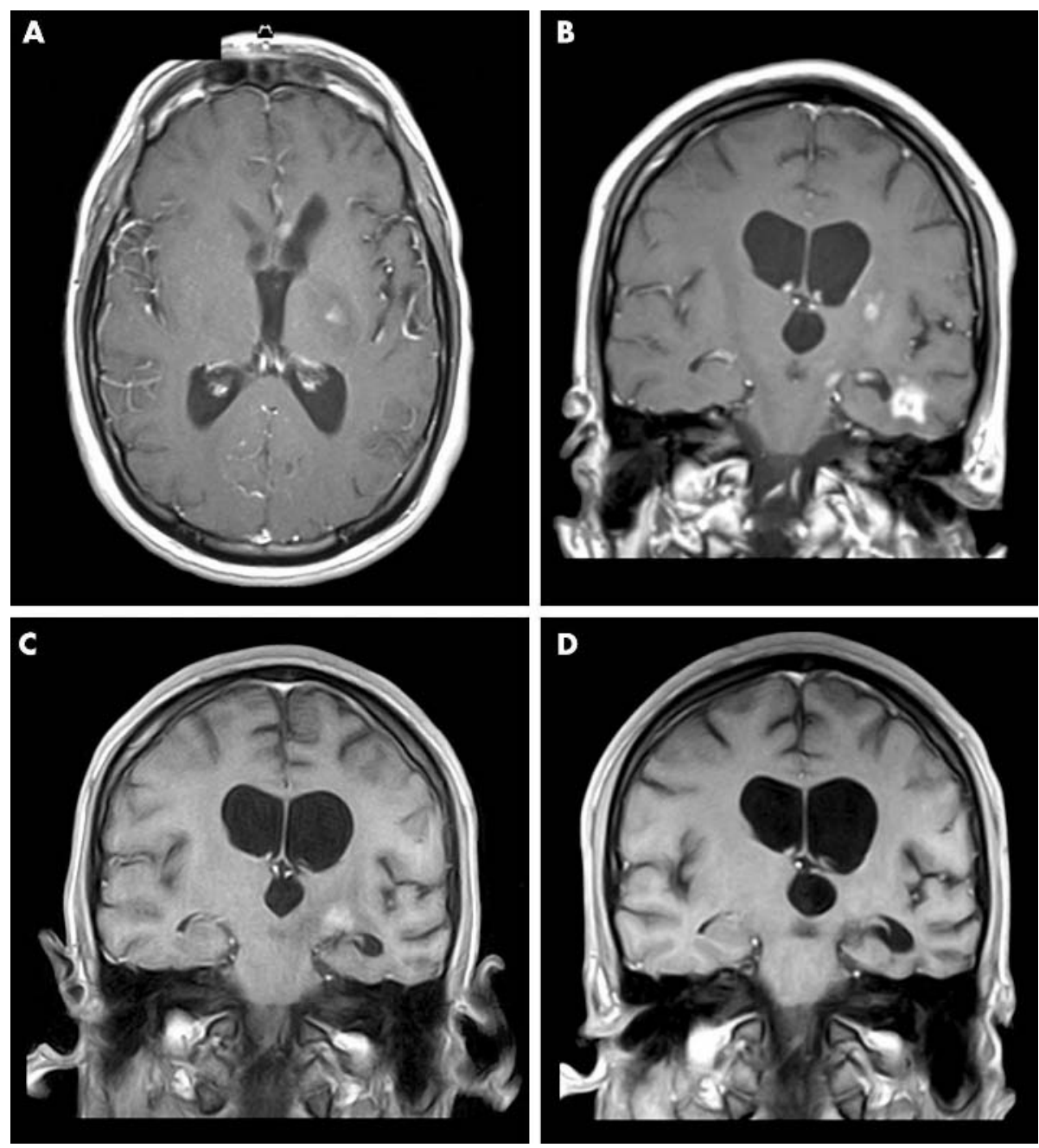

Figure 1 Magnetic resonance images in chronological order. (A) Axial FSE T1 weighted image revealing a lesion of the left posterior internal capsule enhanced after gadolinium injection extending (not shown) into the left thalamus and mesencephalon. Note also the left callosal ischaemic lesion. (B) Contrast enhanced coronal FSE T1 weighted image 5 months later demonstrating reappearance of capsular and mesencephalic lesions as well as a new left temporal lesion. (C) Coronal FSE TI weighted image 2 years later showing new contrast enhancement of the left posterior internal capsule. (D) Recent coronal FSE T1 weighted image showing no more contrast enhancing lesions. Note the subcortical atrophy.

choice. CSF analysis ranges from major pleocytosis with predominantly polymorphonuclear cells in the acute stage to slight pleocytosis with normal protein and glucose level in chronic parenchymal disease.

TNF is a potent pro-inflammatory cytokine. Its importance in the pathogenesis of several inflammatory disorders has been demonstrated, including BD. Levels of circulating TNF $\alpha$ and soluble TNF receptor are increased in the peripheral blood of patients with active BD. ${ }^{3}{ }^{4}$ There is experimental evidence of enhanced TNF $\alpha$ production by monocytes and $\gamma \delta \mathrm{T}$ cells in patients with BD. Both spontaneous and lipopolysaccharide stimulated TNF $\alpha$ production is higher in $\mathrm{BD}$ than in control individuals. ${ }^{5-7}$ It is not clear if the inappropriate regulation of $\operatorname{TNF} \alpha$ in $\mathrm{BD}$ is genetically determined, although TNF promoter polymorphism has been demonstrated in some patients. ${ }^{8}$ The central role of TNF $\alpha$ in the pathogenesis of BD is further supported by an increasing number of reports highlighting the efficacy of anti-TNF $\alpha$

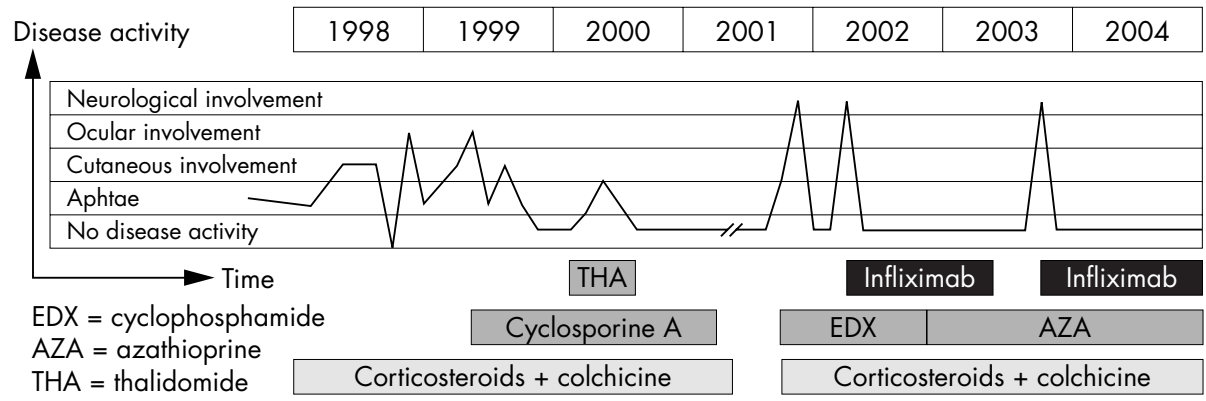

Figure 2 Graph showing disease activity and drugs administered over time. 
agents in this disease. According to a recent review of published and unpublished data, both infliximab and etanercept (a soluble TNF receptor) have been successfully used to treat different manifestations of BD. ${ }^{9}$ Etanercept $25 \mathrm{mg} \mathrm{s}$ /c biweekly has proven efficacy in suppressing mucocutaneous manifestations in 40 male patients in a Turkish, double blind, placebo controlled study. Recurrence was observed in some patients 3 months after etanercept discontinuation. ${ }^{9}$ Etanercept added to conventional immunosuppressant agents in 10 patients with refractory uveitis had a beneficial effect in maintaining visual acuity and allowed a decrease in corticosteroid dosage during the 6 month treatment period. However, this effect was lost after etanercept discontinuation. ${ }^{9}$ Infliximab has been successfully used for severe mucocutaneous, ocular, and intestinal manifestations of $\mathrm{BD}$ refractory to conventional treatment. The anti-inflammatory effect was strong and of rapid onset.

Potential side effects of TNF $\alpha$ blockade are hypersensitivity reactions, fever, chills, increased frequency of infections including reactivation of tuberculosis, and lupus-like disease. In addition, reports of demyelinating disease in rheumatoid arthritis and Crohn's disease as well as exacerbation of multiple sclerosis under TNF $\alpha$ blockade are of particular interest and concern. ${ }^{10}$ Several mechanisms have been proposed to explain the auto-immune features associated with TNF $\alpha$ blockade. ${ }^{10}$ Indeed, systemic administration of anti-TNF $\alpha$ may increase autoimmunity by inhibiting apoptosis of autoreactive $\mathrm{T}$ cells, increasing $\mathrm{T}$ cell receptor signalling, and interfering with antigen presenting cell functions. Thus, TNF $\alpha$ may be seen as a double edged sword: when at high levels it may mediate pathology leading to tissue injury, while at low levels it may be needed to inhibit autoimmune manifestations. Why infliximab is effective in NB remains unknown since the blood-brain barrier (BBB) may hamper its access to cerebral parenchyma. It can be hypothesised, however, that the anti-inflammatory effect of TNF $\alpha$ antagonists in the central nervous system depends on the degree of disruption of the BBB, as shown in experimental autoimmune encephalomyelitis.

Formation of antibodies to infliximab is an emerging problem. These antibodies are associated with infusion reactions and loss of initial response. Concurrent immunosuppressive therapy reduces formation of antibodies to infliximab. ${ }^{11}$

To the best of our knowledge there is only one additional clinical report on the use of an anti-TNF $\alpha$ agent in NB. ${ }^{12} \mathrm{~A}$ 59 year old woman with $\mathrm{BD}$ and cerebral parenchyma involvement relapsed under treatment with corticosteroids and cyclophosphamide. Upon addition of infliximab $5 \mathrm{mg} / \mathrm{kg}$ at 0,2 , and 6 weeks, rapid resolution of neurological symptoms and radiological signs were observed. There was no evidence of active disease 2 months after the last infusion. In our case also, treatment with infliximab resulted in rapid resolution of radiological signs, and bimonthly infusions for 12 month not only prevented relapses but allowed a considerable decrease in concomitant immunosuppressant medication. The protective effect, however, was not sustained and the relapse observed 7 months after cessation is a strong indication that TNF $\alpha$ blockade in BD has only a suppressive activity and that long term treatment should be considered.

In conclusion, infliximab seems to be effective in several manifestations of $\mathrm{BD}$, including involvement of the central nervous system parenchyma. Relapses may occur after cessation of anti-TNF blockade. Further studies are needed to determine the long term efficacy and safety of this treatment.

\section{Authors' affiliations}

C Ribi, C Chizzolini, Clinical Immunology and Allergy, Department of Internal Medicine, University Hospital Geneva, Geneva, Switzerland R Sztajzel, Department of Neurology, University Hospital Geneva, Geneva, Switzerland

J Delavelle, Department of Radiology, University Hospital Geneva, Geneva, Switzerland

Competing interests: none declared

The patient detailed in this study agreed to his details being published.

Correspondence to: Camillo Ribi, Clinical Immunology and Allergy, Department of Internal Medicine, University Hospital Geneva, Geneva, Switzerland; camillo.ribi@hcuge.ch

Received 6 October 2004

Revised version received 8 March 2005

Accepted 25 March 2005

\section{REFERENCES}

1 Siva A, Kantarci OH, Saip S, et al. Behçet's disease: diagnostic and prognostic aspects of neurological involvement. J Neurol $2001 ; 248(2): 95-103$.

2 Kidd D, Steuer A, Denman AM, et al. Neurological complications in Behçet's syndrome. Brain 1999;122(Pt 11):2183-94.

3 Turan B, Gallati H, Erdi H, et al. Systemic levels of the T cell regulatory cytokines IL-10 and IL-12 in Behçet's disease: soluble TNFR-75 as a biological marker of disease activity. J Rheumatol 1997;24(1):128-32.

4 Evereklioglu C, Er H, Turkoz Y, et al. Serum levels of TNF-alpha, slL-2R, IL-6, and IL-8 are increased and associated with elevated lipid peroxidation in patients with Behçet's disease. Mediators Inflamm 2002;1 1(2):87-93.

5 Mege JL, Dilsen N, Sanguedolce V, et al. Overproduction of monocyte derived tumor necrosis factor alpha, interleukin (IL) 6, IL-8 and increased neutrophil superoxide generation in Behçet's disease. A comparative study with familial Mediterranean fever and healthy subjects. J Rheumatol 1993;20(9):1544-9.

6 Yamashita N, Kaneoka H, Kaneko S, et al. Role of gammadelta T lymphocytes in the development of Behçet's disease. Clin Exp Immunol 1997; 107(2):241-7.

7 Freysdottir J, Lau S, Fortune F. Gammadelta T cells in Behçet's disease (BD) and recurrent aphthous stomatitis (RAS). Clin Exp Immunol 1999;1 18(3):451-7

8 Ahmad T, Wallace GR, James T, et al. Mapping the HLA association in Behçet's disease: a role for tumor necrosis factor polymorphisms? Arthritis Rheum 2003;48(3):807-13.

9 Sfikakis PP. Behçet's disease: a new target for anti-tumour necrosis factor treatment. Ann Rheum Dis 2002;61(Suppl 2):ii51-3.

10 Robinson WH, Genovese MC, Moreland LW. Demyelinating and neurologic events reported in association with tumor necrosis factor alpha antagonism: by what mechanisms could tumor necrosis factor alpha antagonists improve rheumatoid arthritis but exacerbate multiple sclerosis? Arthritis Rheum 2001;44(9): 1977-83.

11 Farrell RJ, Alsahli M, Jeen YT, et al. Intravenous hydrocortisone premedication reduces antibodies to infliximab in Crohn's disease: a randomized controlled trial. Gastroenterology 2003;124(4):917-24.

12 Licata G, Pinto A, Tuttolomondo A, et al. Anti-tumour necrosis factor alpha monoclonal antibody therapy for recalcitrant cerebral vasculitis in a patient with Behçet's syndrome. Ann Rheum Dis 2003;62(3):280-1. 